\title{
A SiPM-Based Clinical MRI-Compatible SPECT Insert
}

\author{
M. Carminati, Senior Member, IEEE, I. D’Adda, A. J. Morahan, K. Erlandsson, K. Nagy, Z. Nyitrai, M. Czeller, \\ R. Moresco, A. Savi, P. Van Mullekom, B. F. Hutton, Senior Member, IEEE and C. Fiorini, Senior Member, IEEE
}

\begin{abstract}
The first SiPM-based clinical SPECT ring for brain simultaneous imaging in standard MR scanners has been put into operation. Reliable operation of the instrument has been achieved by means of its optimization, such as the introduction of a dual-cooling system (liquid-gas) of the DAQ electronics. The static gantry is composed of 20 MRI-compatible $\gamma$-detection modules mounting $5 \mathrm{~cm} \times 10 \mathrm{~cm}$ CsI(TI) scintillators for a total of 1440 pixels (targeting a transaxial $\mathrm{FoV}$ of $20 \mathrm{~cm}$ ). Here we present the results of a preliminary characterization showing $220 \mathrm{kcps}$ max. count rate, $365 \mathrm{cps} / \mathrm{MBq}$ sensitivity with the multislit-slat collimator, $\sim 17 \%$ energy resolution and an average extrinsic spatial resolution of $\sim 8 \mathrm{~mm}$ in the trans-axial plane.
\end{abstract}

\section{INTRODUCTION}

$\mathrm{W}$ ITHIN the rapidly expanding domain of multimodal imaging, the integration of SPECT and MRI is lagging with respect to PET/MRI [1]. However, SPECT can provide broader versatility than PET [2]. In order to tackle this delay, mostly due to MRI-compatibility issues of the collimator [3] and detector scalability issues, we present the first clinical insert for simultaneous SPECT/MRI of the human brain, targeting, in particular, neuro-oncology [4]. This instrument represents the scaled-up version of a preclinical insert [5] and it is based on large arrays of SiPMs (1440 pixels in total). Preliminary results in terms of basic SPECT performance are here presented, while a more extended characterization is reported in [6-7].

\section{INSTRUMENT DESIGN}

Since the rotation of the detectors inside the MR scanner is not allowed, the SPECT insert is composed of 20 gamma cameras mounted on a static plastic gantry. As a result of the compromise between size and spatial resolution, the ring is not complete, presenting a gap in the bottom part. As shown in Fig. 1, the internal bore is $35 \mathrm{~cm}$ wide (without the customdesigned clinical RF coil), and the external dimensions are

Manuscript received December 13, 2019. This work was supported by the EU under project INSERT (grant \# 305311) and also by the EPSRC (grant \# EP/R511638/1) and the NIHR UCLH Biomedical Research Centre.

M. Carminati, I. D'Adda, C. Fiorini are with Dipartimento di Elettronica, Informazione e Bioingegneria, Politecnico di Milano, Milano 20133, Italy and with INFN, Sez. di Milano, Milano 20133 Italy (e-mail: marcol.carminati@polimi.it).

A. J. Morahan, K. Erlandsson, and B. F. Hutton are with University College London, Institute of Nuclear Medicine, London NW1 2BU, UK.

K. Nagy, Z. Nyitrai, M. Czeller are with MEDISO Ltd., Budapest, 1037 Hungary.

A. Savi and R. Moresco are with San Raffaele Scientific Institute, Milano, 20132 Italy.

P. Van Mullekom is with Nuclear Fields, 5827 AP Vortum-Mullem, The Netherlands.
$56 \mathrm{~cm}$ (horizontal) by $44 \mathrm{~cm}$ (vertical) and the total weight is about $50 \mathrm{~kg}$. The collimator has been designed as an innovative multimini slit-slat type [8-9] and it has been fabricated as a composition of about 6000 insulated slices in order to reduce Eddy currents. The target field of view (FoV) is $20 \mathrm{~cm}$ (transaxial) by $9 \mathrm{~cm}$ (axial).

The instrument architecture is summarized in Fig. 2. In addition to the insert, placed inside an unmodified MR scanner, the main power supply, the Ethernet gateway interfacing with the optical daisy chain and the chiller (used to operate to the detectors at $-10^{\circ} \mathrm{C}$ ) are placed in the control room.

Each detection module is based on a continuous $\mathrm{CsI}(\mathrm{Tl})$ scintillator $(50 \mathrm{~mm} \times 100 \mathrm{~mm} \times 8 \mathrm{~mm})$ coupled to 8 tiles of SiPM (RGB-HD by FBK) whose current signals are read by two custom-designed 36-channel ASICs (named ANGUS [10]). Data acquisition is performed by local FPGA modules in daisy-chain (Fig. 2), providing optical interconnection to a main gateway in the control room. Event localization is carried out by means of statistical methods (MLEM).

The detection modules [11], all the electronics and the RF coils have been already individually characterized, especially in terms of mutual compatibility with MRI [12]. However, the

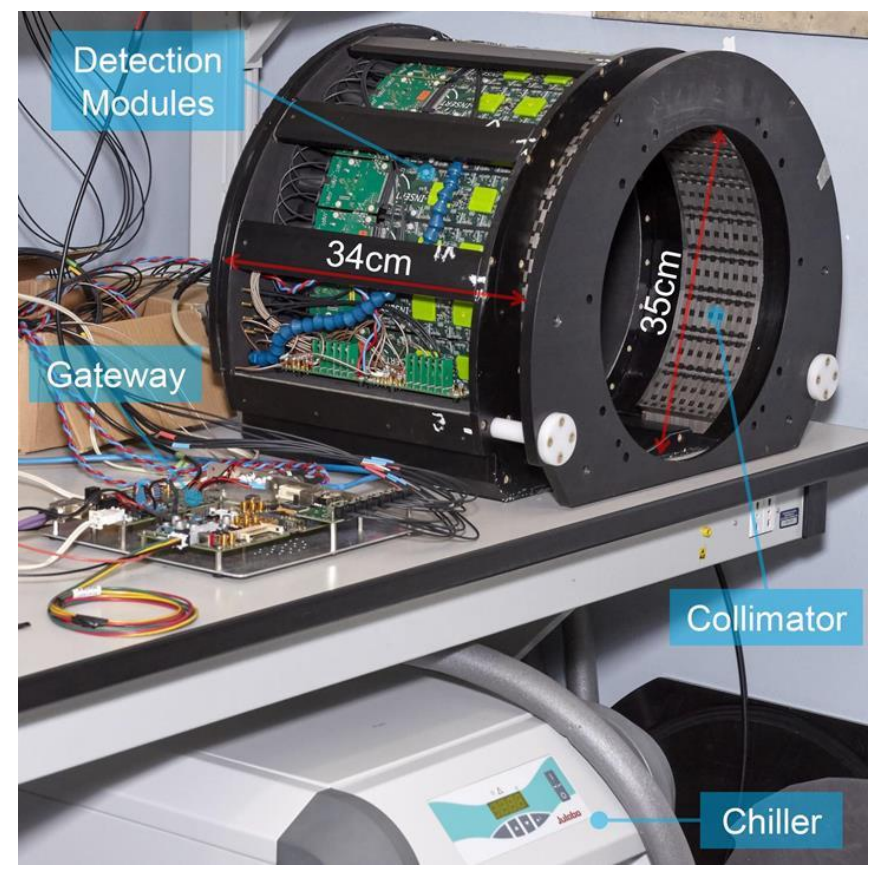

Fig. 1. Fully assembled clinical SPECT scanner featuring 20 detection modules with $5 \mathrm{~cm} \times 10 \mathrm{~cm} \mathrm{CsI(Tl)} \mathrm{scintillators} \mathrm{coupled} \mathrm{to} \mathrm{SiPMs} \mathrm{(for} \mathrm{a} \mathrm{total}$ of 1440 pixels). 


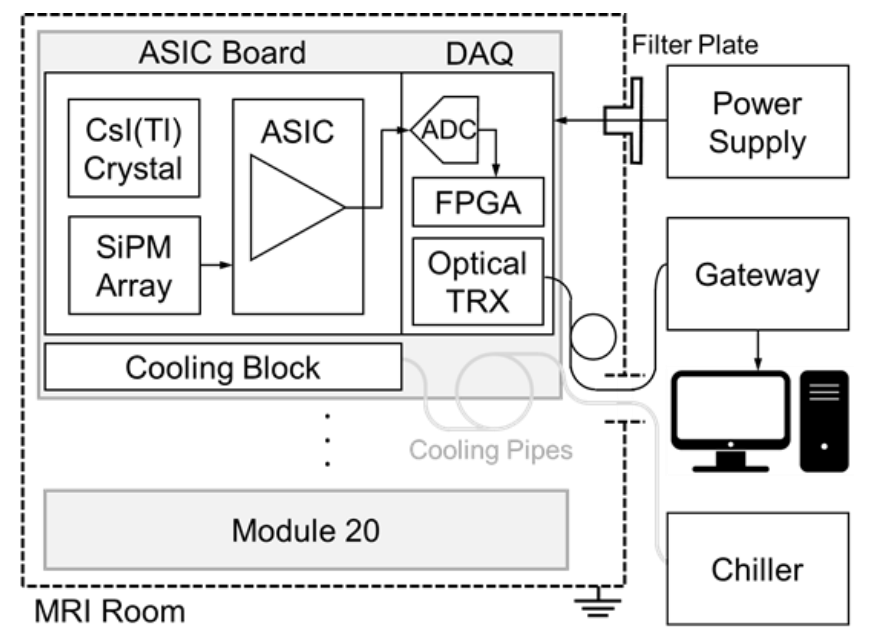

Fig. 2. Architecture of the instrument electronics portioned among the analog detector board and the DAQ one with optical digital communication. Pipes, optical fibers and power supply cables are 15 -m long.

impact of the collimator on MRI is still to be assessed. The preclinical predecessor (with a $50 \mathrm{~mm} \times 50 \mathrm{~mm}$ crystal and a full ring of 10 modules with a transaxial FoV of $15.6 \mathrm{~mm}$ ) had shown no performance degradation due to simultaneous operation in a 3T-scanner (12\% energy resolution at $140 \mathrm{keV}$, $1 \mathrm{~mm}$ intrinsic resolution, $0.9 \mathrm{~mm}$ extrinsic resolution, and $1100 \mathrm{cps} / \mathrm{MBq}$ sensitivity for a multi-pinhole collimator [7]).

An issue due to overheating of the DAQ boards had emerged in the clinical instrument. It has been solved by the introduction of a gas cooling and recirculation circuit. The nitrogen cooling is derived from the main liquid recirculation system cooling the detectors at $-10^{\circ} \mathrm{C}$ (Fig. 3).

\section{EXPERIMENTAL RESULTS}

\section{A. Count Rate}

Count rate tests were performed by preparing several point sources of ${ }^{99 \mathrm{~m}} \mathrm{Tc}$ with different activities, measured at different times along the decay. The dead time of the processing

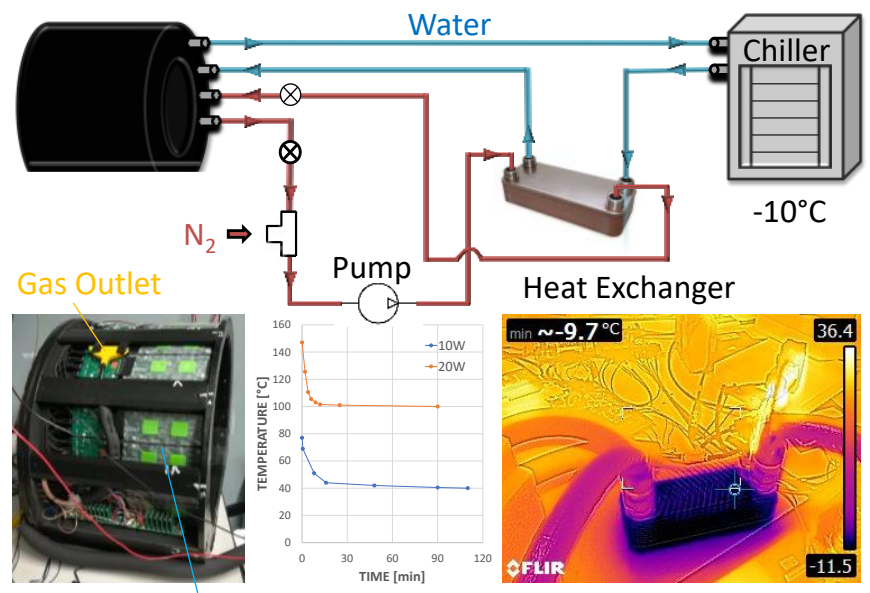

Anti-condensation Wrapping

Fig. 3. Chiller-based (Julabo FL1204) dual MRI-compatible cooling approach introducing cold $\mathrm{N}_{2}$ recirculation to avoid overheating of the DAQ boards (far from the detector 3D-printed cooling blocks).
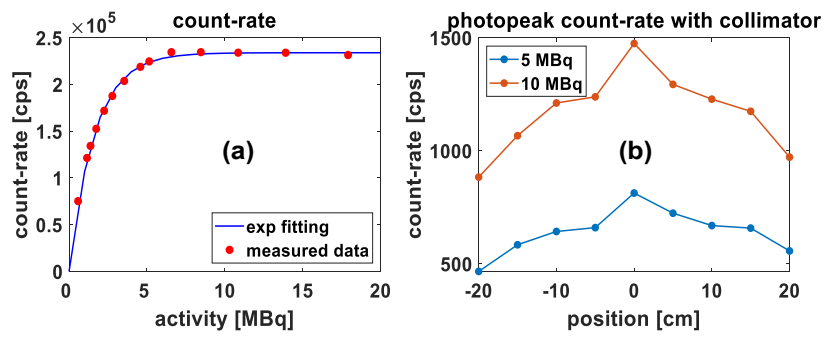

photopeak count-rate with collimator

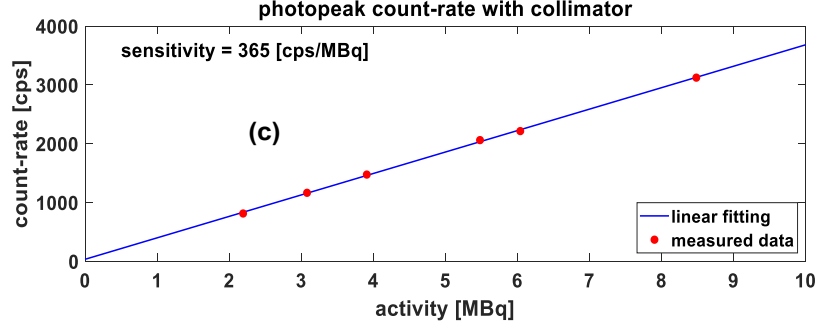

Fig. 4. Count rate characterization at different ${ }^{99} \mathrm{~m}$ Tc activities: (a) max. count rate without collimator, (b) count-rate dependence with respect to position and (c) sensitivity with the multimini-slit-slat collimator.

electronics, including both the analog RC shaping time $(7.5 \mu \mathrm{s})$ and acquisition time, sets a: maximum count rate of $\sim 11 \mathrm{kcps}$ for each module, corresponding to a global limit of $\sim 220 \mathrm{kcps}$. As reported in Fig. 4a, this corresponds to a saturation in the number of counted events for activities above $5 \mathrm{MBq}$ without the collimator. When the collimator is in place (Fig. 3b), the number of collected events drops down, allowing the operation of activities in the FoV up to $\sim 100 \mathrm{MBq}$. In the linear region of the response with the collimator (Fig. 3c), a sensitivity of $365 \mathrm{cps} / \mathrm{MBq}$ has been estimated. This value is consistent with simulations [8].

\section{B. Energy Resolution}

Energy calibration has been performed both by using multiple radiotracers as well as with a single one $\left({ }^{99 \mathrm{~m}} \mathrm{Tc}\right)$ and subtracting the offset of the readout by means of on-board injection capacitors than can pulse the ASIC and acquisition chain. The two calibration techniques proved to be equivalent. Figure 5 reports the measured energy spectra for all the modules with a ${ }^{99 \mathrm{~m}} \mathrm{Tc}$ vial without (a) and with (b) the collimator. The energy resolution, computed after corrections of the planar image (linearity and uniformity correction, rejection of the events whose reconstructed coordinates are at the edges of the FoV in a 5mm-thick frame), is reported in Fig. 6. It also shows the impact of extracting the optical model (a Gaussian light response function LRF) from a flood illumination in the same operating conditions). The average energy resolution is $\sim 17 \%$ at $140 \mathrm{keV}$. It is worse than previous measurements [11]. The large variability among the modules suggests that there might be a deterioration of the crystal, of the optical resin or of the SiPMs.

\section{Spatial Resolution}

Finally, glass capillaries with an inner diameter below $1 \mathrm{~mm}$ were used to assess the spatial resolution. For a single module, the measured FWHM as a function of the distance of the capillary from the collimator surface is reported in Fig. 7. 
(a) No Collimator
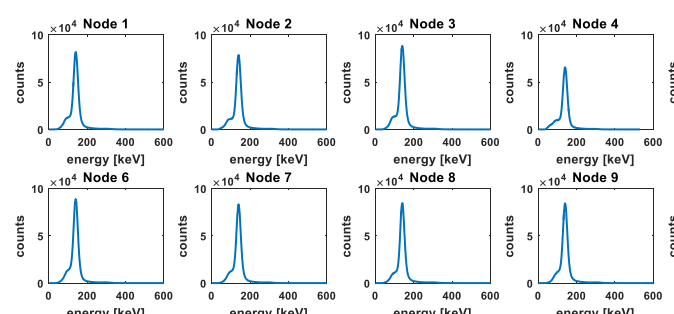

energy
$\times 10^{4}$ Node 11
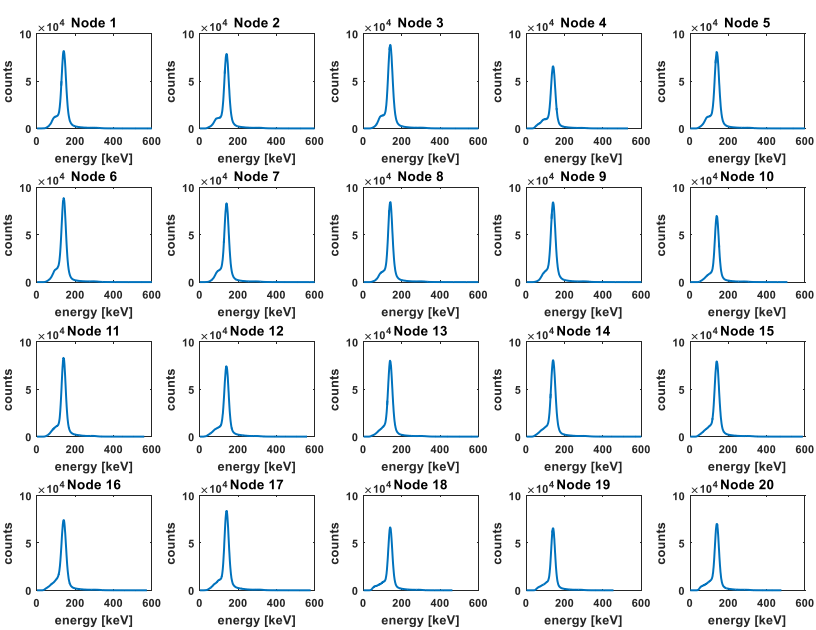
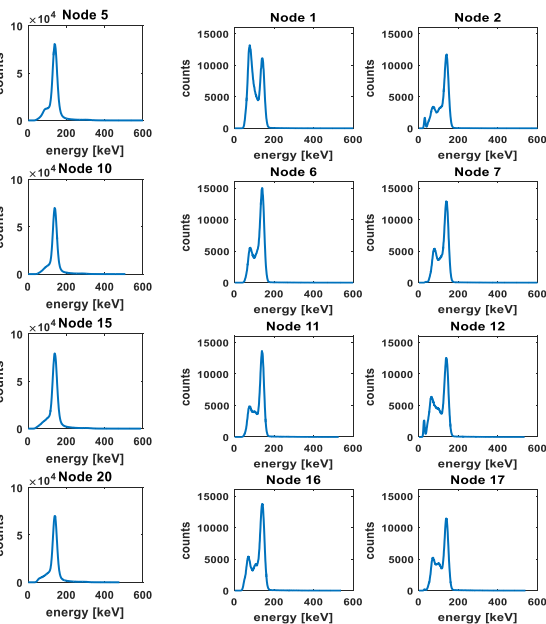

(b) With Collimator

Fig. 5. Acquired spectra from all 20 modules with a ${ }^{99 m} \mathrm{Tc}$ source both without (a) and with (b) the collimator.

The planar spatial resolution here obtained is consistent with intrinsic spatial resolution of the module $(\sim 1 \mathrm{~mm},[11])$ and with the collimator minification ( 8, [9]). The tomographic reconstruction of the capillaries, shows an average extrinsic resolution of about $8 \mathrm{~mm}$ [6-7], thus matching the target of $10 \mathrm{~mm}$.

\section{CONCLUSIONS}

The scalability of SiPM detection modules to clinical scanners has been demonstrated together with the long-term stability of the instrument. Thanks to a modular design of the MRI-compatible electronics, the growth in complexity from a preclinical to a clinical design mostly impacts on thermal and mechanical aspects which have been successfully addressed.

\section{ACKNOWLEDGMENT}

All the students, researchers and partner institutions supporting the INSERT project, especially beyond its formal end, are warmly acknowledged, in particular: M. Occhipinti, F. Baratelli and D. Salvado.

\section{REFERENCES}

[1] S. Vandenberghe and P. K Marsden, "PET-MRI: a review of challenges

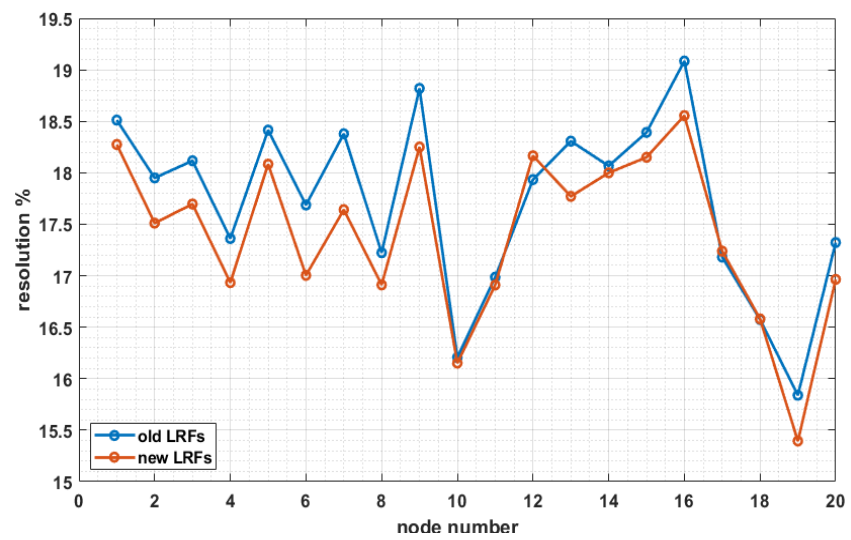

Fig. 6. Energy resolution at $140 \mathrm{keV}$ extracted from the spectra of Fig. $5 \mathrm{~b}$ after correction of linearity and uniformity of the reconstructed floods. and solutions in the development of integrated multimodality imaging," Phys. Med. Biol. vol. 60, pp. R115-R154, 2015.

[2] M.R. Bernsen, P. E. B. Vaissier, R. Van Holen, J. Booij, F. J. Beekman, M. de Jong, "The role of preclinical SPECT in oncological and neurological research in combination with either CT or MRI," Eur. J. Nucl. Med. Mol. Imaging, vol. 41, pp. S36-S49, 2014.

[3] K. Van Audenhaege et al., "Review of SPECT collimator selection, optimization, and fabrication for clinical and preclinical imaging," Med. Phys., vol. 42, no. 8, pp. 4796-4813, Aug. 2015.

[4] B. Hutton et al., "Development of clinical simultaneous SPECT/MRI," Brit. J. Radiology, vol. 90, $20160690,2017$.

[5] M. Carminati et al., "Validation and Performance Assessment of a Preclinical SiPM-Based SPECT/MRI Insert," IEEE Trans. Rad. Plasma Med. Sci., vol. 3, no.4, pp. 483-490, July 2019.

[6] A. J. Morahan et al, "Acquisition Correction and Reconstruction for a Clinical SPECT/MRI Insert," in Proc. IEEE NSS/MIC 2019, in press.

[7] M. Carminati et al., "Clinical SiPM-Based MRI-Compatible SPECT: Preliminary Characterization," IEEE Trans. Rad. Plasma Med. Sci., in press.

[8] D. Salvado et al., "Collimator design for a brain SPECT/MRI insert," IEEE Trans. Nucl. Sci., vol. 62, no. 4, pp. 1716-1724, 2015.

[9] D. Salvado et al., "Development of a Practical Calibration Procedure for a Clinical SPECT/MRI System Using a Single INSERT Prototype Detector and Multimini Slit-Slat Collimator," IEEE Trans. Rad. Plasma Med. Sci., vol. 2, no. 4, pp. 380-386, July 2018.

[10] P. Trigilio, P. Busca, R. Quaglia, M. Occhipinti and C. Fiorini, "A SiPM-readout ASIC for SPECT applications," IEEE Trans. Rad. Plasma Med. Sci., vol. 2, no. 5, pp. 404-410, 2018.

[11] M. Occhipinti et al., "Characterization of the detection module of the insert SPECT/MRI clinical system," IEEE Trans. Rad. Plasma Med. Sci., vol. 2, no. 6, pp. 554-563, Nov. 2018.

[12] M. Carminati et al., "SPECT/MRI INSERT compatibility: assessment, of the Detection Module of the INSERT SPECT/MRI Clinical System" IEEE Trans. Rad. Plasma Med. Sci., vol. 2, no. 6, pp. 554-563, 2018.
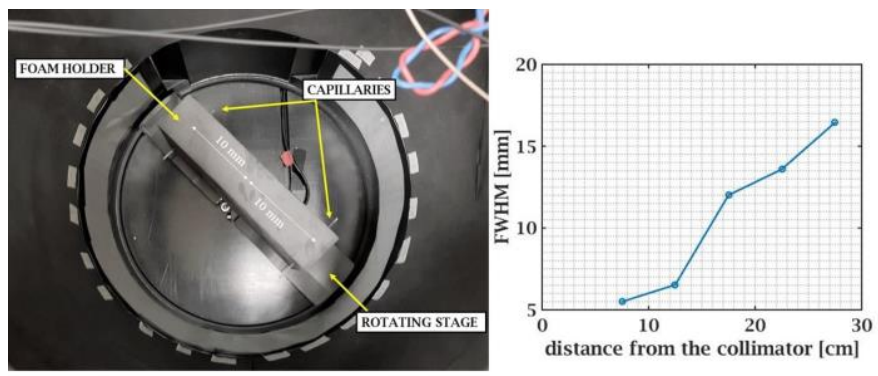

Fig. 7. Spatial resolution (averaged among 5 modules) as a distance of the capillary from the collimator surface. 